Proc. of the 15th Int. Workshop on Slow Positron Beam Techniques and Applications, Prague, September 2-6, 2019

\title{
Construction of a Spin-Polarized Positronium Time-of-Flight Measurement Apparatus
}

\author{
M. Maekawa*, K. Wada, A. Miyashita and A. Kawasuso \\ Quantum Beam Science Research Directorate, \\ National Institutes for Quantum and Radiological Science and Technology, \\ 1233 Watanuki, Takasaki, Gunma, 370-1292, Japan
}

\begin{abstract}
For the measurement of the energy-resolved spin polarization of the outermost surface of materials, a spinpolarized positronium time-of-flight spectrometer has been developed. For the non-magnetic material (Pt film) and ferromagnetic material ( $\mathrm{Ni}$ film), spin-polarized positronium time-of-flight measurements were carried out. Although no significant intensity appears for the $\mathrm{Pt}$ sample, a clear negative intensity is observed around the Ps work function energy for the Ni sample. This means that the spin polarization of the outermost electron is negative near the Fermi energy. The spin-polarized positronium time-of-flight method would be a unique tool in the measurement of spin-polarized band structure of outermost electrons due to its energy resolvability and surface selectivity.
\end{abstract}

DOI: 10.12693/APhysPolA.137.105

PACS/topics: spin-polarized positron beam, positronium time-of-flight, electron spin polarization, Fermi level

\section{Introduction}

For the study of novel surface spin phenomena, such as the spin-Hall effect [1], the Rashba effect [2], and topological insulators [3], it is necessary to accurately determine the electron spin polarization on the outermost surface of materials. Especially, the spin polarization near the Fermi level is very important to evaluate the spin transportation. Spin- and angle-resolved photoelectron spectroscopy (SARPES) is one of the powerful techniques at present. However, it is difficult to obtain information only from the surface electrons, since the SARPES data contain information for several atomic layers from the surface. A new method having a selectivity for the outermost electrons is required. The spin-polarized Ps timeof-flight method (SP-PsTOF) will respond to such offers.

Using spin-polarized positronium (Ps) annihilation spectroscopy, the electron spin polarization of the outermost layer can be determined, because Ps is only formed at the vacuum side of the surface with the well-suppressed electron density $[4,5]$. We have observed the current-induced spin polarization $[6,7]$ on the total three-photon annihilation fraction of Ps given by

$$
F_{\mathrm{Ps}}^{3 \gamma}=\varepsilon_{1}\left(F_{|11\rangle}+F_{|1-1\rangle}\right)+\varepsilon_{0}\left(F_{|10\rangle}\right),
$$

where $\varepsilon_{1}$ and $\varepsilon_{0}$ are the detection efficiencies of annihilation photons from the $m= \pm 1$ and $m=0$ states, respectively. The spectrum asymmetry

$$
A=\left(F_{+}-F_{-}\right) /\left(F_{+}+F_{-}\right)
$$

is rewritten by

$$
A=\left(2 \varepsilon_{1}-\varepsilon_{0}\right) /\left(2 \varepsilon_{1}+\varepsilon_{0}\right) P_{+} P_{-},
$$

\footnotetext{
*corresponding author; e-mail: maekawa.masaki@qst.go.jp
}

where the $F_{+}$and $F_{-}$are the $o$-Ps annihilation fractions in the positive and negative magnetic fields, $P_{+}$and $P_{-}$ are positron spin polarization and electron spin polarization, respectively [8]. Since $\varepsilon_{1}$ and $\varepsilon_{0}$ are constants [9] and the positron spin polarization $P_{+}$is known, the electron spin polarization $P_{-}$can be determined from the asymmetry of the $o$-Ps intensity with the alternating external magnetic field.

So far, such measurements have been conducted without both energy and angular resolvabilities of Ps. If the spin-polarized Ps annihilation spectroscopy technique has an energy resolution of the emitted Ps, one can obtain the spin-polarized electron density of states (DOS) associated with the outermost layer. When the Ps work function $\left(\phi_{\mathrm{Ps}}\right)$ is negative, $\mathrm{Ps}_{\mathrm{s}}$ is emitted spontaneously with the energy of $E_{\mathrm{Ps}}=\left(E-E_{\mathrm{F}}\right)-\phi_{\mathrm{Ps}}$, W here $E_{\mathrm{F}}$ is the Fermi level and $E$ is the energy of the electron used for Ps formation [10]. Therefore, the energy distribution of emitted Ps reflects the electron DOS near the Fermi level. Especially, the fastest Ps is derived from an electron at the Fermi level. Ps energy can be measured by the time-of-flight method. In this study, we have developed the SP-PsTOF apparatus employing the spin-polarized positron beam and demonstrate that the spin polarization at the Fermi surface of the outermost electron can be measured.

\section{Construction of apparatus}

Figure 1 shows an overview of the SP-PsTOF apparatus developed here. A $440 \mathrm{MBq}^{22} \mathrm{Na}$ source and a copper cone are mounted on a $4 \mathrm{~K}$ refrigerator. A solid krypton moderator is formed on the inner surface of the copper cone and on the source window [11]. A slow positron beam with an energy of $6 \mathrm{keV}$ generated by a modified 


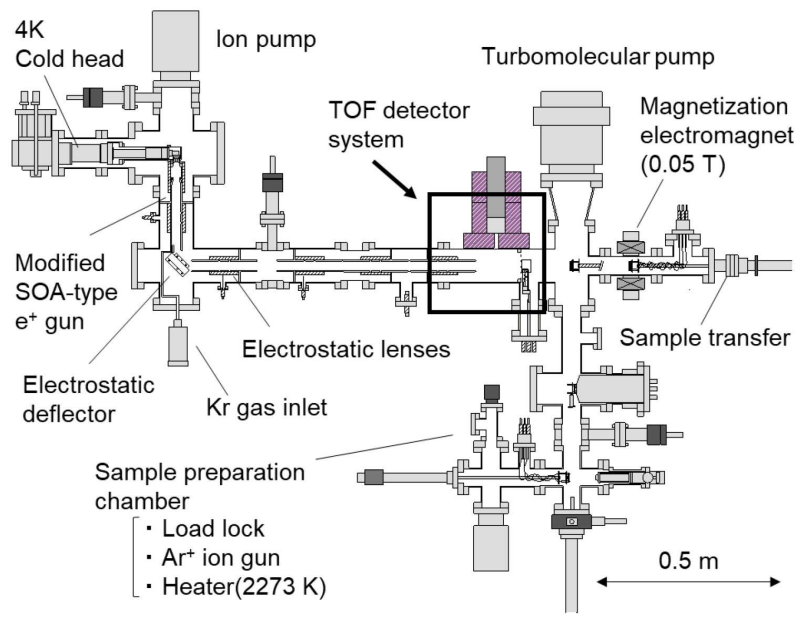

Fig. 1. The schematics of the SP-PsTOF apparatus developed in this work.

SOA-gun [12] is transported by five electrostatic lenses to avoid spin depolarization. An electrostatic deflector turns the longitudinally spin-polarized beam into the transversely spin-polarized beam [13]. Spin polarization was estimated before to be approximately $27 \%$ using the magnetic quenching method [14-16]. Samples are introduced through a preparation chamber, which is equipped with an $\mathrm{Ar}^{+}$sputtering ion gun and a sample heating stage. After the magnetization by an electrostatic magnet, the sample is transferred to the TOF measurement section where a channeltron and $\mathrm{NaI}$ detectors are installed.

Figure 2 shows a schematic diagram of the TOF measurement system. The incident positron beam energy can be controlled in the range of $0.05-6 \mathrm{keV}$ by applying a retarding bias to the sample. To generate start signals from the secondary electrons, a channeltron module (Photonis, model 4831) is installed in front of the sample. Since it is also biased to the same retarding voltage to the sample, signals are taken out through a high-voltage fast capacitor. To collect secondary electrons efficiently, the space in front of channeltron is shielded with a tungsten mesh and aluminum shroud for avoiding dissipation of the secondary electrons by a fringing field. Furthermore, an additional bias $(+50 \mathrm{~V})$ is applied on the channeltron entrance. Due to this bias potential, trajectory of incident positrons is also slightly shifted, however, it is negligible because the positron beam energy is higher enough than that of secondary electrons [17].

Positronium emitted into the vacuum due to the negative work function of several $\mathrm{eV}$ annihilates at several centimeters away from the sample surface. In our apparatus, a lead slit with a width of $15 \mathrm{~mm}$ and a thickness of $50 \mathrm{~mm}$ is installed at $107.5 \mathrm{~mm}$ in front of the sample. The annihilation gamma rays (i.e, stop signal) passing through this slit are detected by three NaI detectors, which are cylindrical with a diameter of $50 \mathrm{~mm}$ and a thickness of $50 \mathrm{~mm}$. Start and stop signals are

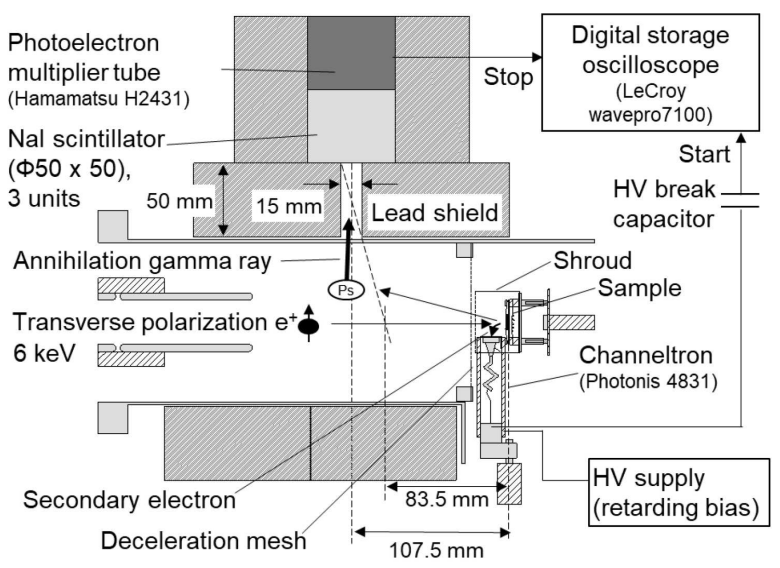

Fig. 2. Schematics of the Ps-TOF measurements system.

collected by a digital oscilloscope providing the Ps timeof-flight spectrum. In most Ps-TOF apparatus [18-20], angle-limiting slits are installed to increase energy resolution. In our apparatus, such a slit is not installed to increase detection efficiency.

\section{TOF measurements}

Test measurements were carried out. The beam intensity was estimated to be $2 \times 10^{5} \mathrm{e}^{+} / \mathrm{s}$ at the sample position and final counting rate of TOF measurement was $\approx 1$ cps. Each spectrum contains more than $3 \times 10^{4}$ events.

Two types of samples were tested. The first one is a non-magnetic sample; Pt film on a sapphire substrate with a size of $10 \times 15 \mathrm{~mm}^{2}$ and a thickness of $200 \mathrm{~nm}$. Figure 3 a shows TOF spectra with the positron beam energy $E=2 \mathrm{keV}$. The intense peak at $t=0$ corresponds to the two-gamma annihilation at the sample (prompt peak). With the sample cleaning by $\mathrm{Ar}^{+}$ion sputtering and heating, the peak intensity around 100 to $400 \mathrm{~ns}$ increased. This indicates that the Ps amount increases as the sample surface becomes cleaner. Figure $3 \mathrm{~b}$ shows the net TOF spectrum obtained by subtracting backgrounds, such as an $o$-Ps decay at the sample and a random constant component. The Ps peak arises at $t=115 \mathrm{ns,}$ which corresponds to the annihilation time of the fastest $o$-Ps at the position closest to the sample in the Ps detection region $\left(L_{\min }=83.5 \mathrm{~mm}\right)$. From the equation $E_{\max }=m / e\left(L_{\min } / t\right)^{2}$, where $m$ is the electron rest mass, $e$ is the elementary electric charge, $L_{\min }$ is the length of the minimum flight path, and $E_{\max }$ is the maximum PS emission energy, $E_{\max }$ (i.e., Ps work function $\phi_{\mathrm{Ps}}$ ) is estimated to be $3.0 \mathrm{eV}$. In general, the Ps work function is given by $\phi_{\mathrm{Ps}}=\phi_{-}-\phi_{+}+E_{b}$, where $E_{b}(6.8 \mathrm{eV})$ is the Ps binding energy [21], $\phi_{-}$and $\phi_{+}$are the electron and positron work functions, respectively. Using reported values $\phi_{-}=5.65 \mathrm{eV}[22]$ and $\phi_{+}=-1.9 \mathrm{eV}[23], \phi_{\mathrm{Ps}}$ for $\mathrm{Pt}$ is calculated to be $2.8 \mathrm{eV}$, which agrees with the experimental value. 


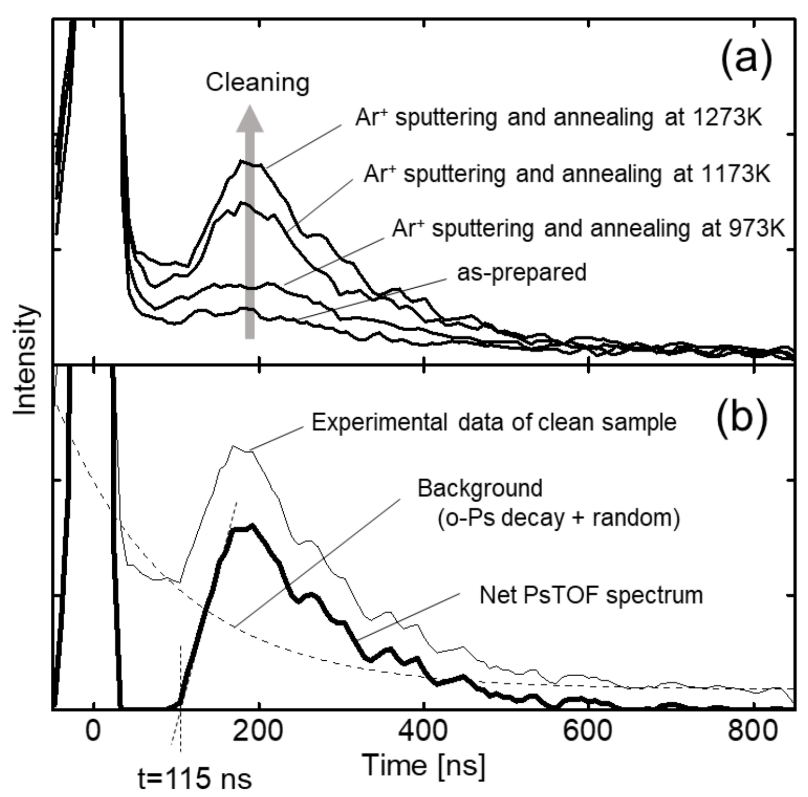

Fig. 3. (a) PsTOF spectra for Pt film sample with the positron beam energy $E=2 \mathrm{keV}$. (b) The net PsTOF spectrum obtained by subtracting backgrounds.
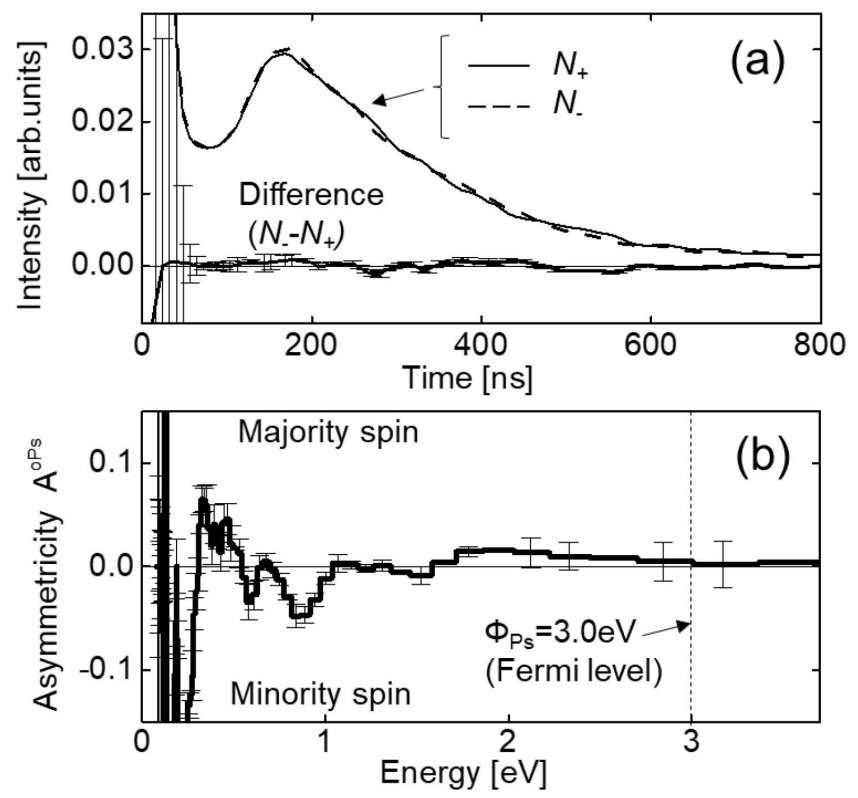

Fig. 4. (a) PsTOF spectra with magnetizing the sample in the positive and negative magnetic field $\left(N_{+}\right.$and $N_{-}$) and its intensity difference $\left(N_{-}-N_{+}\right)$for the $\mathrm{Pt}$ sample. (b) The SP-PsTOF spectrum obtained by converting the intensity difference into the asymmetry.

Figure 4a shows the TOF spectra with the sample magnetized in the positive magnetic field $N_{+}$and in the negative magnetic field $N_{-}$. The difference intensity $\left(N_{-}-N_{+}\right.$, the direction of the external magnetic field is opposite to the direction of the electron spins in material) is also shown. By converting this intensity difference

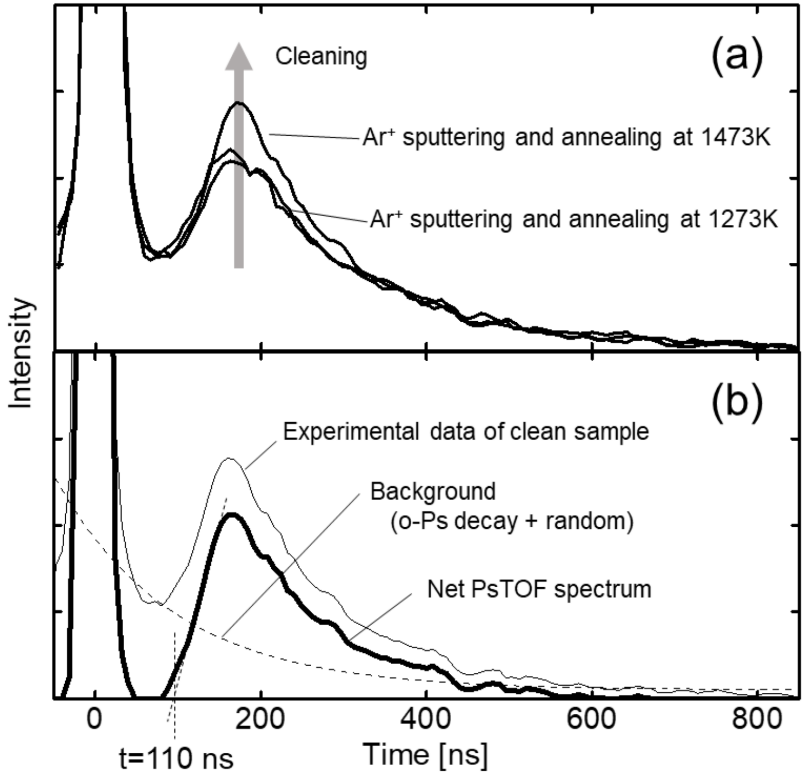

Fig. 5. (a) PsTOF spectra for Ni film sample with the positron beam energy $E=2 \mathrm{keV}$. (b) The net PsTOF spectrum obtained by subtracting backgrounds.

into the asymmetry, $A=\left(N_{-}-N_{+}\right) /\left(N_{-}+N_{+}\right)$, the SP-PsTOF spectrum was obtained as shown in Fig. $4 \mathrm{~b}$. The asymmetry at the Ps work function $\phi_{\mathrm{Ps}}=3.0 \mathrm{eV}$ is almost zero. Because the $\phi_{\mathrm{Ps}}$ (fastest Ps energy) is derived from the electrons at the Fermi level and Ps is still generated only with the outermost electrons, this result indicates that the spin polarization of electrons near the Fermi level at the Pt outermost surface is almost zero.

The second sample is a nickel film, which is a typical ferromagnetic material. Figure 5a shows the Ps-TOF spectra of the Ni sample with the incident positron energy $E=2 \mathrm{keV}$. Just like the Pt sample, the Ps components at around $t=200$ ns are increased by sample cleaning. The net spectrum after subtracting the backgrounds is shown in Fig. 5b. From the rise time of the spectrum $t=110 \mathrm{~ns}$, the work function of Ps is estimated to be $3.2 \mathrm{eV}$, which is in agreement with the reported values [24].

Figure $6 \mathrm{a}$ shows the intensity difference between the positive and negative magnetic fields and the SP-PsTOF spectrum is also shown in Fig. 6b. Unlike Pt sample, the asymmetry at around $\phi_{\mathrm{Ps}}=3.2 \mathrm{eV}$ is clearly observed. This indicates that the spin polarization of electrons on the Ni outermost surface is negative near the Fermi level (minority spin is dominant). Even if the material is ferromagnetic, the surface electrons do not necessarily have the same spin polarization as the bulk electrons. From the theoretical calculations, the electron spin polarization of nickel surface is expected to be negative near the Fermi level [25, 26]. Similar tendency has been reported in SARPES measurements [27]. However, since SARPES data contains information for several layers from the surface, it may not be the polarization of only the outermost 

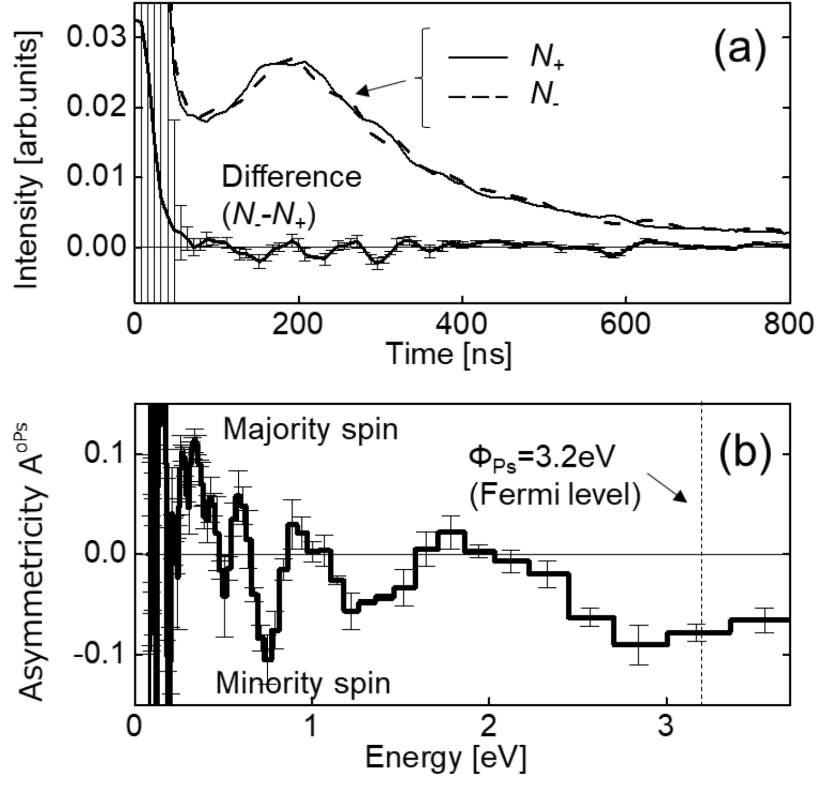

Fig. 6. (a) PsTOF spectra with magnetizing the sample in the positive and negative magnetic field $\left(N_{+}\right.$and $\left.N_{-}\right)$and its intensity difference $\left(N_{-}-N_{+}\right)$for the $\mathrm{Ni}$ sample. (b) The SP-PsTOF spectrum obtained by converting the intensity difference into the asymmetry.

surface electrons. On the other hand, the electrons detected by the SP-PsTOF method are guaranteed to belong at the outermost surface since Ps is generated only with electrons in a low electron density region expanding further from the surface to the vacuum. By the SP-PsTOF method, it is confirmed for the first time that the electron spin polarization of $\mathrm{Ni}$ outermost surface is negative near the Fermi level. The SP-PsTOF method would be a progressive tool for the measurement of spinpolarized band structure of outermost electrons.

\section{Summary}

As the new tool for the evaluation of spin polarization electron DOS having a selectivity only for the outermost electrons, SP-PsTOF apparatus has been developed employing the spin-polarized positron beam. For the non-magnetic $(\mathrm{Pt})$ and ferromagnetic $(\mathrm{Ni})$ sample, the SP-PsTOF measurements were carried out. Although no significant intensity appears near the Fermi level for the $\mathrm{Pt}$ sample, the SP-PsTOF spectrum for the Ni sample shows a clear negative value near the Fermi level, which indicates that the spin polarization of electrons near the Fermi level of the Ni outermost surface is negative. Since the Ps formation is guaranteed to be occurring only with the outermost surface electrons, the SP-PsTOF method might be most powerful tool for the study of the spinpolarized band structure of surface electrons, such as the spin-Hall effect, the Rashba effect, and topological insulators.

\section{References}

[1] M.I. Dyakonov, V.I. Perel, Phys. Lett. A35, 459 (1971).

[2] J. Sinova, D. Culcer, Q. Niu, N.A. Sinitsyn, T. Jungwirth, A.H. MacDonald, Phys. Rev. Lett. 92, 126603 (2004).

[3] C.L. Kane, E.J. Mele, Phys. Rev. Lett. 95, 146802 (2005).

[4] A.P. Mills Jr., Phys. Rev. Lett. 41, 1828 (1978).

[5] A. Ishii, Solid State Phenom. 28, 213 (1992).

[6] A. Kawasuso, Y. Fukaya, M. Maekawa, H.J. Zhang, T. Seki, T. Yoshino, E. Saitoh, K. Takanashi, J. Magn. Magn. Mater. 342, 139 (2013).

[7] H.J. Zhang, J.S. Yamamoto, Y. Fukaya, M. Maekawa, H. Li, A. Kawasuso, T. Seki, E. Saitoh, K. Takanashi, Sci. Rep. 4, 4844 (2014).

[8] D.W. Gidley, A.R. Köymen, T.W. Capehart, Phys. Rev. Lett. 49, 1779 (1982).

[9] R.M. Drisko, Phys. Rev. 102, 1542 (1956).

[10] A. Ishii, S. Shindo, Surf. Sci. 242, 256 (1991).

[11] A.P. Mills Jr., S.S. Voris Jr., Tamara S. Andrew, J. Appl. Phys. 76, 2556 (1994).

[12] F.J. Mulligan, M.S. Lubell, Meas. Sci. Technol. 4, 197 (1993).

[13] S.S. Hanna, R.S. Preston, Phys. Rev. 106, 1363 (1957).

[14] Y. Nagai, Y. Nagashima, J. Kim, Y. Itoh, T. Hyodo, Nucl. Instrum. Methods Phys. Res. B 171, 199 (2000).

[15] M. Maekawa, Y. Fukaya, A. Yabuuchi, I. Mochizuki, A. Kawasuso, Nucl. Instrum. Methods Phys. Res. B 308, 9 (2013).

[16] M. Maekawa, Y. Fukaya, H. Zhang, H. Li, A. Kawasuso, J. Phys. Conf. Ser. 505, 012033 (2014).

[17] M. Maekawa, K. Wada, A. Miyashita, A. Kawasuso, J. Phys. Conf. Ser. 791, 012009 (2017).

[18] A.P. Mills, Jr., L. Pfeiffer, P.M. Platzman, Phys. Rev. Lett. 51, 1085 (1983).

[19] R.H. Howell, I.J. Rosenberg, M.J. Fluss, Phys. Rev. B 34, 3069 (1986).

[20] H. Terabe, S. Iida, T. Yamashita, T. Tachibana, B. Barbiellini, K. Wada, I. Mochizuki, A. Yagishita, T. Hyodo, Y. Nagashima, Surf. Sci. 641, 68 (2015).

[21] A. Kawasuso, M. Maekawa, A. Miyashita, K. Wada, T. Kaiwa, Y. Nagashima, Phys. Rev. B 97, 245303 (2018).

[22] H.B. Michaelson, J. Appl. Phys. 48, 4729 (1977).

[23] P.G. Coleman, in: Positron Beams and Their Application, World Sci., 2000, p. 136.

[24] R.H. Howell, I.J. Rosenberg, M.J. Fluss, R.E. Goldberg, R.B. Laughlin, Phys. Rev. B 35, 5303(R) (1987).

[25] Y.S. Dedkov, M. Fonin, New J. Phys. 12, 12 (2010).

[26] K. Wada, A. Miyashita, M. Maekawa, S. Sakai, A. Kawasuso, AIP Conf. Proc. 1970, 040001 (2018).

[27] C.H. Park, B.C. Lee, J.I. Lee, J. Kor. Phys. Soc. 47, 655 (2005). 